\title{
Remote Sensing Image Captioning with Continuous Output Neural Models
}

\author{
Rita Ramos \\ INESC-ID \\ Instituto Superior Técnico, University of Lisbon \\ Lisbon, Portugal \\ rita.mparada.ramos@gmail.com
}

\author{
Bruno Martins \\ INESC-ID \& LUMLIS (Lisbon ELLIS Unit) \\ Instituto Superior Técnico, University of Lisbon \\ Lisbon, Portugal \\ ritaparadaramos@tecnico.ulisboa.pt
}

\begin{abstract}
Remote sensing image captioning involves generating a concise textual description for an input aerial image. Most previous methods are based on neural encoder-decoder models trained to generate a sequence of discrete outputs with the standard cross-entropy token-level loss. This paper explores an alternative method based on continuous outputs, generating sequences of embedding vectors instead of directly predicting discrete word tokens. We argue that continuous outputs can facilitate the optimization of semantic similarity, as opposed to exact word-by-word matches. It also facilitates the use of loss functions that compare different views of the data. This includes comparing representations for individual tokens and for the entire captions, and also comparing captions against intermediate image representations. We experimentally compared discrete versus continuous output methods over the RSICD dataset, extensively used in the area. Results show that continuous outputs can indeed lead to better results, and our approach performs competitively with the state-of-the-art model in the area.
\end{abstract}

\section{CCS CONCEPTS}

- Computing methodologies $\rightarrow$ Machine learning; Natural language processing; Neural networks.

\section{KEYWORDS}

Remote Sensing Image Captioning, Natural Language Generation, Deep Neural Networks

ACM Reference Format:

Rita Ramos and Bruno Martins. 2021. Remote Sensing Image Captioning with Continuous Output Neural Models. In 29th International Conference on Advances in Geographic Information Systems (SIGSPATIAL '21), November 2-5, 2021, Beijing, China. ACM, New York, NY, USA, 4 pages. https://doi. org $/ 10.1145 / 3474717.3483631$

\section{INTRODUCTION}

Automatically annotating remote sensing images with short text descriptions (i.e., captions) can be an effective approach to describe the contents of large image repositories concerning specific areas of the Earth. The generated captions can be used to find and group

Permission to make digital or hard copies of part or all of this work for personal or classroom use is granted without fee provided that copies are not made or distributed for profit or commercial advantage and that copies bear this notice and the full citation on the first page. Copyrights for third-party components of this work must be honored

For all other uses, contact the owner/author(s).

SIGSPATIAL '21, November 2-5, 2021, Beijing, China

(C) 2021 Copyright held by the owner/author(s).

ACM ISBN 978-1-4503-8664-7/21/11.

https://doi.org/10.1145/3474717.3483631 similar images through textual queries, thus rendering large remote sensing image collections as both indexable and discoverable.

The predominant approach for this task involves the use of encoder-decoder neural models proposed for general image captioning [10]. Previous methods have mostly differed in the attention mechanisms that are considered [6]. In these approaches, a convolutional encoder is typically used to build representations for the different regions of an input image. These representations are then used by a recurrent decoder that generates the caption word-byword, at each step using neural attention to weight the contribution of the different image regions, according to relevance for the current prediction. The model is trained with the standard cross-entropy loss, predicting word tokens through a softmax normalization layer that generates a probability distribution over the vocabulary. A decoding algorithm (e.g., greedy decoding or a beam search procedure) can then sample from the resulting probability distribution, in order to autoregressively generate the output caption.

Recently, an alternative approach for language generation was suggested by Kumar and Tsvetkov [5] in the context of machine translation, replacing the conventional softmax layer with an embedding layer (i.e., replacing the generation of discrete token outputs with the generation of continuous vector representations). Training efficiency was the main motivation behind continuous output models. Specifically, the original authors wanted to alleviate the burden of using a softmax layer, which becomes prohibitively expensive as the vocabulary size increases, often leading to the practical choice of sacrificing part of the vocabulary (e.g., rare words).

In our work, we argue that there are other advantages to generation with continuous outputs. In particular, these approaches can facilitate the use of novel loss functions to optimize semantic similarity at different granularities: token level, sentence level, and in terms of image similarity. We propose a novel image captioning method that uses continuous outputs in the decoder component, also featuring other differences to previous approaches (e.g., using an EfficientNet model [11] as the image encoder, fine-tuned for remote sensing images). Our main contributions are as follows:

- We propose a novel encoder-decoder framework for remote sensing image captioning, using continuous outputs in the decoder, together with a strong image encoder pre-trained with in-domain data, that also predicts continuous representations through natural language supervision.

- We designed novel loss functions for model training with basis on continuous outputs, going beyond token-level optimization and integrating different components.

- We advanced a novel decoding strategy that also explores continuous outputs, going beyond standard beam search by 
evaluating the generated sentences according to similarity towards the input image.

- We released our code ${ }^{1}$ to advance and encourage future research on remote sensing image captioning.

\section{THE PROPOSED APPROACH}

The traditional approach for language generation is based on discrete outputs, using a softmax normalization layer to produce, for each output position, a probability distribution over the tokens in a vocabulary (i.e., for assigning scores to discrete tokens). The alternative generation method based on continuous outputs [5] instead employs a final layer that produces embedding vectors, replacing the generation of discrete tokens for the generation of word embeddings. Thus, continuous output models can be optimized in respect to semantic similarity, e.g. by minimizing the distance between the output embedding and the target word embedding, as opposed to optimizing exact word-by-word matches with the cross-entropy loss. We further argue that continuous outputs can facilitate model training in ways that potentiate the use of novel loss functions, e.g. comparing the generated captions at the sequence-level (instead of, or in complement to, performing comparisons at the level of individual tokens) against the ground-truth captions, or comparing the generated captions against representations for the input images [7]. In our work, we combine the use of continuous outputs for language generation, leveraging different and complementary loss terms, together with a novel encoding procedure for remote sensing images, which also produces continuous representations through natural language supervision.

\subsection{The Image Encoder Component}

Most remote sensing image captioning studies use a CNN encoder pre-trained on the ImageNet dataset, although ImageNet only contains natural images (i.e., ground-level photos) with very different characteristics from remote sensing images [6]. Additionally, the ImageNet task involves classifying the main object of the image, whereas for remote sensing image captioning there are often multiple objects of interest, that need to be considered within a single image. This mismatch between the type of input images and task/domain can make the image representations, produced with standard encoders, less effective. In our work, we fine-tune a $\mathrm{CNN}$ encoder on the remote sensing image captioning datasets, considering a fine-tuning task based on natural language supervision related to predict embeddings from associated captions. This training procedure is in line with recent studies that efficiently learn image representations from natural language [9].

Typically, the encoder in previous studies is a ResNet model pre-trained to predict discrete image classes, using a final softmax normalization layer to predict a probability distribution over the possible classes. We instead employ an EfficientNet model [11] that that is instead trained to predict an embedding for a reference caption associated to the input image. First, we assign each image in the training data with a target embedding built from a reference caption, by randomly sampling from the multiple reference captions associated to each image and then averaging the corresponding GloVe word embeddings. Then, the last fully-connected

\footnotetext{
${ }^{1}$ https://github.com/RitaRamo/remote-sensing-images-caption/tree/master/src
}

layer from the EfficientNet model, initially pre-trained on ImageNet, is replaced by a different fully-connected layer with dimensionality equal to 300 (i.e., the dimensionality of the GloVe embeddings [8] used in our experiments). The resulting model is fine-tuned with the smooth L1 loss, computed between the predicted caption embedding and the target caption embedding. The smooth L1 loss, also referred to as the Huber loss, produces a linear penalty when the absolute difference between predictions and targets is high, and a quadratic penalty when the difference is close to zero. The equation for comparing two values $x$ and $y$ is as follows and, when comparing embedding vectors, we can take the average over the different vector dimensions.

$$
\operatorname{smoothL1}(x, y)= \begin{cases}\frac{1}{2}(x-y)^{2} & \text { for }|x-y| \leq 1 \\ |x-y|-\frac{1}{2}, & \text { otherwise }\end{cases}
$$

\subsection{Generation with Continuous Outputs}

Our language decoder corresponds to a Long Short-Term Memory (LSTM) unit [4] followed by a fully-connected layer that outputs a continuous embedding, in replacement of the standard fullyconnected vocabulary projection layer with softmax normalization. In this way, the model generates embeddings, rather than probability distributions over the token vocabulary.

The decoder is also combined with a neural attention mechanism, in which a visual context vector for each generation step $t$ is computed with a scaled dot-product attention [12], given the image features $V$ (i.e., a set of vector representations for $K$ different image regions) and the previous hidden state $h_{t-1}$ of the LSTM:

$$
c_{t}=\sum_{i=1}^{K} \alpha_{i, t} v_{i}, \quad \text { with } \quad \alpha_{t}=\operatorname{softmax}\left(\frac{V^{T} W h_{t-1}}{\sqrt{d}}\right) .
$$

In the previous expression, $W$ is a learned parameter tensor, $d$ is the dimensionality of the image features $V, \alpha_{t}$ are the attention weights for each image region, and $c_{t}$ is the attended image vector (i.e., the resulting visual context vector, corresponding to a weighted average of the image features $v_{i}$ for the $K$ different regions).

For each input image, the EfficientNet encoder extracts representative features, and the initial hidden states of the LSTM are then initialized with a global mean-pooled image feature, projected to the same dimensionality of the LSTM hidden states. At each timestep, the LSTM decoder receives as input the GloVe embedding of the current word, concatenated with the visual attention context vector. It then predicts an embedding word vector as output.

The captioning model is trained with a novel loss function that was specifically designed to explore generation with continuous outputs. This function combines 3 separate terms: (a) a token-level smooth L1 loss computed between predicted and target embeddings; (b) a sentence-level smooth L1 loss computed between a representation for the generated caption (i.e., the mean of the predicted embeddings) and the ground-truth caption (i.e., the mean of the target embeddings); (c) an image-level loss comparing the generated caption against the image [7], corresponding to the smooth L1 loss between the average of the predicted embeddings, and the image representation obtained from the last layer of the encoder.

$$
L_{1}=\operatorname{smoothL1}\left(\hat{e_{t}}, e_{t}\right),
$$




$$
\begin{gathered}
L_{2}=\operatorname{smoothL1}\left(\frac{1}{T} \sum_{t=1}^{T} \hat{e_{t}}, \frac{1}{T^{\prime}} \sum_{t=1}^{T^{\prime}} e_{t}\right), \\
L_{3}=\operatorname{smoothL} 1\left(\frac{1}{T} \sum_{t=1}^{T} \hat{e_{t}}, i\right), \\
\text { loss }=w_{1} L_{1}+w_{2} L_{2}+w_{3} L_{3} .
\end{gathered}
$$

In the previous expressions, $\hat{e}_{t}$ is the predicted embedding at timestep $t, e_{t}$ is the corresponding target embedding, and $i$ is the image input representation. Each of the 3 separate terms (i.e., $L_{1}, L_{2}$, and $L_{3}$ ) has a weight parameter to be adjusted, respectively corresponding to $w_{1}, w_{2}$, and $w_{3}$ (see Section 3.1 for training details).

At inference time, we can use the greedy nearest-neighbor decoding scheme proposed by Kumar and Tsvetkov [5] for continuous outputs. In this method, a word is generated from a predicted embedding by selecting the word of the vocabulary that has the nearest embedding, according to the smooth L1 loss (i.e., the minimum value according to the function described in Equation 1).

Besides greedy decoding, we also tested an adapted beam-search procedure that runs $b$ parallel searches through the sequences of most likely words, leveraging continuous outputs as a way to score the entire generated captions according to similarity towards the input image. In our tailored decoding procedure, at each generation step, a beam (i.e., a sequence of words generated up to a given step) is evaluated with basis on (a) the similarity of the embedding evaluated by the model at the current step and the corresponding target word embedding, and (b) the similarity between the average of the embeddings for the words generated thus far and the current embedding, evaluated against the image representation.

$$
\text { score }=\lambda \cdot \operatorname{smoothL} 1\left(\hat{e_{T}}, w_{T}\right)+(1-\lambda) \cdot \operatorname{smoothL} 1\left(\frac{1}{T} \sum_{t=1}^{T} w_{t}, i\right) .
$$

In the previous expression, $\hat{e}_{t}$ is the predicted word embedding at time-step $T, w_{T}$ is the embedding for the word being evaluated as a candidate for step $T, i$ is the image representation, and $\lambda$ is a tuning parameter controlling the contribution of the similarity towards the image.

\section{EXPERIMENTAL EVALUATION}

This section presents the experimental evaluation of the proposed method, detailing the experimental protocol and the results.

\subsection{Dataset and Methods}

We report experimental results on the commonly used RSICD dataset, which is currently the largest publicly available dataset in the area. We specifically used the revised version from $\mathrm{Li}$ et al. [6], which fixed some typographical and grammatical errors. The dataset contains 10921 images with 30 different categories. Each image has $224 \times 224$ pixels with different ground-level resolutions, and is associated to 5 reference captions, resulting in a total of 54605 captions. We used the original training, validation, and test splits $(80 \%, 10 \%$, and $10 \%$ of the images, respectively), and we also used the available pre-tokenized words as the vocabulary.

To evaluate the proposed approach, we used standard metrics in the literature for assessing text generation methods [2], including BLEU (i.e., BLEU-1, BLEU-2, BLEU-3, and BLEU-4), ROUGE_L,
METEOR, CIDEr, SPICE, and the recently proposed BERTScore metric [13]. All the aforementioned metrics, with the exception of BERTScore $^{2}$, were calculated through the implementation in the MS-COCO caption evaluation package ${ }^{3}$.

For the image encoder, we used an EfficientNet-B5 model ${ }^{4}$, pretrained on ImageNet and fine-tuned for predicting embeddings for captions. The fine-tuning used $90 \%$ of the RSICD training images, and the remaining $10 \%$ were left out for adjusting early stopping (i.e., to stop training after 5 consecutive epochs without improvement). We used the Adam optimizer with an initial learning rate of $1 \mathrm{e}-4$, adjusting the parameters of all the layers (i.e., each EfficientNet layer is unfreezed). A set of simple data augmentation operations was considered in the fine-tuning of the encoder, implemented through the Albumentations library [1]. In particular, each image is augmented with a geometric transformation (e.g., rotations or flips) or with a color perturbation (50\% chance, respectively).

On what regards the decoder, the LSTM hidden state has a dimensionality of 512 , followed by a dropout layer of 0.5 that precedes the output layer. Both the LSTM decoder and the EfficientNet encoder produce outputs with a dimensionality of 300 , corresponding to the size of the pre-trained GloVe embeddings [8] that are used as targets. The attention context vector has also 300 dimensions.

For training the complete model, we used Adam with a learning rate of $4 \mathrm{e}-4$, only adjusting the parameters of the decoder (i.e., the weights of the encoder are fixed) and relying on a teacher forcing strategy. The learning rate is decayed after 5 consecutive epochs without improvement (with a shrink factor of 0.8 ), and the training is stopped if there is no improvement after 12 consecutive epochs. The loss weighting terms (i.e., $w_{1}, w_{2}$ and $w_{3}$ ) were learned with GradNorm [3], i.e. an algorithm to automatically balance the contribution of different components/tasks within a loss function, that works on the basis of dynamically tuning the gradient magnitudes. We training the model once with GradNorm, and then used the discovered weights $(1.18,1.01$, and 0.81 , respectively) to re-train the model. We used Adam for the GradNorm optimizer, with a learning rate of 0.025 . Finally, on what regards the parameters of the beam-search procedure, we use a beam of 5 , with 0.03 for the value of the $\lambda$ parameter in Equation 9, a minimum length of 6 words for the generated captions, and preventing the repetition of the same word more than twice.

\subsection{Experimental Results}

We first attempted to verify the effectiveness of the proposed encoder, comparing a baseline encoder-decoder model with neural attention (i.e., a model using EfficientNet pre-trained on ImageNet as the encoder, together with a standard softmax decoder) against a similar model using the encoder fine-tuned with in-domain data. The results are presented at the top of Table 1, showing that the fine-tuned encoder can clearly outperform the standard encoder.

Next, we focused on assessing the use of continuous outputs. Table 1 (middle) shows the results, using the fine-tuned encoder together with the continuous output decoder. We looked at the contribution of the different loss terms described in Section 2.2. The model trained with the $L_{1}$ loss (i.e., optimizing the similarity

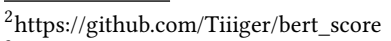

${ }^{3}$ https://github.com/tylin/coco-caption

${ }^{4}$ https://github.com/lukemelas/EfficientNet-PyTorch
} 


\begin{tabular}{|c|c|c|c|c|c|c|c|c|c|}
\hline Methods & BLEU 1 & BLEU 2 & BLEU 3 & BLEU 4 & METEOR & CIDEr & ROUGE_L & SPICE & BERTScore \\
\hline Baseline (w/o fine-tuned encoder) & 0.6355 & 0.5062 & 0.4135 & 0.3430 & 0.3100 & 1.8228 & 0.5639 & 0.3796 & 0.5896 \\
\hline Discrete Model & 0.7495 & 0.6227 & 0.5294 & 0.4544 & 0.3771 & 2.5047 & 0.6619 & 0.4823 & 0.6865 \\
\hline Continuous Model: $L_{1}$ & 0.7535 & 0.6414 & 0.5499 & 0.4735 & $\underline{0.3820}$ & 2.6952 & 0.6753 & 0.4913 & $\underline{0.6966}$ \\
\hline Continuous Model: $L_{1}+L_{2}$ & 0.7711 & 0.6550 & 0.5610 & 0.4852 & $\overline{0.3726}$ & 2.6182 & 0.6684 & 0.4854 & $\overline{0.6902}$ \\
\hline Continuous Model: $L_{1}+L_{2}+L_{3}$ & 0.7676 & 0.6581 & 0.5692 & 0.4961 & 0.3745 & 2.6603 & 0.6712 & 0.4828 & 0.6948 \\
\hline "" $L\left(w_{1}=1.18, w_{2}=1.01, w_{3}=0.81\right)$ & 0.7836 & 0.6751 & 0.5849 & 0.5107 & 0.3738 & 2.7474 & 0.6783 & 0.4854 & 0.7026 \\
\hline "" $L$ w/ Beam Search & $\underline{0.7846}$ & 0.6794 & 0.5915 & 0.5190 & 0.3714 & 2.7777 & $\underline{0.6810}$ & $\underline{0.4891}$ & 0.7073 \\
\hline Previous State-of-the-Art [6] & 0.8058 & $\underline{0.6778}$ & $\underline{0.5866}$ & $\underline{0.5163}$ & 0.4718 & $\underline{2.7716}$ & 0.7237 & 0.4786 & - \\
\hline
\end{tabular}

Table 1: Results on the RSICD dataset. Top: Comparison for the attention-based encoder-decoder model leveraging discrete outputs, without and with the fine-tuned encoder. Middle: The proposed encoder-decoder model based on continuous outputs, i.e. the fine-tuned encoder together with the continuous output decoder, finally combined with beam-search decoding. Bottom: The current state-of-the art model. The best performance is shown in bold and the second-best is underlined.

of each predicted word embedding against the target embeddings) achieves a better performance on all the metrics, when compared to the the conventional model based on discrete outputs, trained with the cross-entropy loss. Adding a sentence-level loss term (i.e., $L_{1}+L_{2}$ ) further improves results for some of the metrics (e.g., BLEU scores), and the same is also true when adding the image-level loss term (i.e., $L_{1}+L_{2}+L_{3}$ ). The best results are obtained from balancing the contribution of each loss term through the use of GradNorm, clearly outperforming the standard approach based on discrete outputs in terms of all the metrics. This includes the metrics that better correlate with human judgements, namely for CIDEr with an improvement of 0.2427 , SPICE with an improvement of 0.0031 , and BERTScore with an improvement of 0.0161 .

We also compared the default greedy decoding approach, against the tailored beam-search procedure that was advanced at the end of Section 3.2. The results show slight improvements with the use of beam-search decoding, over most of the metrics.

Finally, the bottom line in Table 1 shows that the proposed approach is competitive against the current state-of-art, namely the multi-level attention model of Li et al. [6]. Our model achieves slightly higher scores in terms of several metrics, including metrics that better correlate with human judgements (e.g., CIDEr or SPICE). Moreover, our contributions can also be combined with other neural attention mechanisms, similar to those from Li et al. [6].

\section{CONCLUSIONS}

This paper presented a novel encoder-decoder model for remote sensing image captioning. We specifically explored the use of continuous output representations for language generation, replacing the generation of discrete tokens in favour of word embeddings. Experimental results confirmed the effectiveness of the proposed approach. Our tests showed that fine-tuning the encoder on remote sensing images can lead to significant performance improvements, and the alternative generation approach based on continuous outputs can better capture the global semantic similarity between captions and images. The overall results are also comparable with the current state-of-art model in the area.

For future work, we can perhaps consider an alternative decoderdecoder architecture based on the Transformer [12], given that this type of models is currently achieving state-of-the-art results on a variety of language processing and computer vision tasks.

\section{ACKNOWLEDGMENTS}

This research was supported by Fundação para a Ciência e Tecnologia (FCT), through the project grant with reference PTDC/CCICIF/32607/2017 (MIMU) and the PhD scholarship with reference 2020.06106.BD, as well as the INESC-ID multi-annual funding from the PIDDAC programme with reference UIDB/50021/2020. We also gratefully acknowledge the support of NVIDIA Corporation, with the donation of the two Titan Xp GPUs used in our experiments.

\section{REFERENCES}

[1] Alexander Buslaev, Vladimir I Iglovikov, Eugene Khvedchenya, Alex Parinov, Mikhail Druzhinin, and Alexandr A Kalinin. 2020. Albumentations: fast and flexible image augmentations. Information 11, 2 (2020).

[2] Asli Celikyilmaz, Elizabeth Clark, and Jianfeng Gao. 2020. Evaluation of Text Generation: A Survey. arXiv preprint arXiv:2006.14799 (2020).

[3] Zhao Chen, Vijay Badrinarayanan, Chen-Yu Lee, and Andrew Rabinovich. 2018. GradNorm: Gradient normalization for adaptive loss balancing in deep multitask networks. In Proceeedings of the International Conference on Machine Learning.

[4] Sepp Hochreiter and Jürgen Schmidhuber. 1997. Long short-term memory. Neural Computation 9, 8 (1997).

[5] Sachin Kumar and Yulia Tsvetkov. 2018. Von Mises-Fisher loss for training sequence to sequence models with continuous outputs. arXiv preprint arXiv:1812.04616 (2018)

[6] Yangyang Li, Shuangkang Fang, Licheng Jiao, Ruijiao Liu, and Ronghua Shang. 2020. A Multi-Level Attention Model for Remote Sensing Image Captions. Remote Sensing 12, 6 (2020).

[7] Mitja Nikolaus, Mostafa Abdou, Matthew Lamm, Rahul Aralikatte, and Desmond Elliott. 2019. Compositional Generalization in Image Captioning. In Proceedings of the Conference on Computational Natural Language Learning.

[8] Jeffrey Pennington, Richard Socher, and Christopher D. Manning. 2014. GloVe: Global vectors for word representation. In Proceedings of the Conference on Empirical Methods in Natural Language Processing.

[9] Alec Radford, Jong Wook Kim, Chris Hallacy, Aditya Ramesh, Gabriel Goh, Sandhini Agarwal, Girish Sastry, Amanda Askell, Pamela Mishkin, Jack Clark, et al. 2021. Learning transferable visual models from natural language supervision. arXiv preprint arXiv:2103.00020 (2021).

[10] Matteo Stefanini, Marcella Cornia, Lorenzo Baraldi, Silvia Cascianelli, Giuseppe Fiameni, and Rita Cucchiara. 2021. From Show to Tell: A Survey on Image Captioning. arXiv preprint arXiv:2107.06912 (2021).

[11] Mingxing Tan and Quoc Le. 2019. EfficientNet: Rethinking Model Scaling for Convolutional Neural Networks. In Proceedings of the International Conference on Machine Learning.

[12] Ashish Vaswani, Noam Shazeer, Niki Parmar, Jakob Uszkoreit, Llion Jones, Aidan N Gomez, Łukasz Kaiser, and Illia Polosukhin. 2017. Attention is all you need. Proceedings of the Annual Meeting on Neural Information Processing Systems (2017).

[13] Tianyi Zhang, Varsha Kishore, Felix Wu, Kilian Q Weinberger, and Yoav Artzi. 2019. BERTscore: Evaluating text generation with BERT. arXiv preprint arXiv:1904.09675 (2019) 